\title{
Parametric Optimization of a Two Stage Vapor Compression Refrigeration System by Comparative Evolutionary Techniques
}

\author{
Shuhaimi Mahadzir ${ }^{1 *}$ and Rasel Ahmed ${ }^{1}$ \\ ${ }^{1}$ Chemical Engineering Department, Universiti Teknologi PETRONAS, Malaysia \\ Centre for Process Systems Engineering, Institute of Autonomous System, Universiti Teknologi PETRONAS, Malaysia
}

\begin{abstract}
Multistage refrigeration system plays a vital role in industrial refrigeration for the chemical, petrochemical, pharmaceuticals and food industries. Modern chemical industries are complex, and the problems are commonly multi-dimensional, non-linear and time-consuming. This study presents the application of evolutionary computation techniques, namely PSO (particle swarm optimization), GA (Genetic Algorithm) and SA (Simulated Annealing) to solve a design problem of a two-stage vapor compression refrigeration system. Two objectives are evaluated, namely the minimization of total energy consumption and maximization of the coefficient of performance (COP) of the system. The basis of design for the two-stage refrigeration system is built from and validated against data from published literature. The mass flow ratio, evaporator and condenser temperature, parameters for subcooling and desuperheating, and the coefficient of performance for the basis of design show acceptable results. The errors are below $5 \%$ against the data from published literature, which are within errors of significant figures in the calculations. In this work, the optimum solutions show a reduction of the required amount of energy consumption by $30.8 \%$ and an increase of the COP by nearly $77 \%$ with respect to the basis of design. Further improvements are made to the optimization procedures to prevent early convergence and to increase the search efficiency for finding the global optima. The findings by PSO, GA and SA are in agreement, and all evolutionary techniques achieved proper convergence of the two objective functions. It is also found that PSO requires lower computational effort, less computation time and is also easier to implement compared to GA and SA.
\end{abstract}

\section{Introduction}

Vapor compression refrigeration systems (VCRS) are widely used in air-conditioning as well as industrial refrigeration system for the chemical, petrochemical, pharmaceuticals and food processes. Modern life would be unthinkable without refrigeration. Global energy consumption is expected to increase by $50 \%$ between 2010 and 2040 [1], while the refrigeration system is projected to consume approximately $30 \%$ of global electric energy by 2050 [2]. The refrigeration system requires energy input to create cooling by removing heat from a low- temperature reservoir. In this case, optimization has been considered as a significant tool to minimize the required energy and cost as well as maximize the efficiency of industrial refrigeration systems [3],[4].

Zhao et al. (2013) developed a model-based optimization technique for VCRS to minimize total operation cost with respect to the constraints of mechanical limitations, component interactions, environment conditions and cooling load demands [5]. Their proposed algorithm is designed to store the best populations in each generation that satisfy the physical constraints with maximum fitness value for finding a feasible solution. According to their findings, the overall energy savings per day is $8.45 \%$, and they noticed $3.43 \%$ dissimilarity between simulated and experimental results [5]. Arshad and coworkers (2019) used GA to optimize the exergy efficiency, COP and exergy destruction of VCRS considering parallel and series flow configurations. For the optimal operating conditions, COP and exergy efficiency is almost $15.1 \%$ and $1.41 \%$ higher for parallel flow than the series flow configuration [4]. The performance of a two-stage VCRS has been analyzed by Torella [6] and Baakeem et al. [7]. Baakeem performed system optimization using Conjugate Directions Method by maximizing the coefficient of performance (COP). It was possible to enhance $\mathrm{COP}$ by increasing the subcooling parameter, decreasing desuperheating parameter and by using different refrigerants. However, refrigerant $\mathrm{R} 134 \mathrm{a}$ does not produce a good performance with the COP only achieving a maximum of 6.01 at $10^{\circ} \mathrm{C}$ evaporator temperature and $40^{\circ} \mathrm{C}$ condenser temperature, respectively.

\footnotetext{
* Corresponding author: shuham@utp.edu.my
} 
Keywords: Two Stage Refrigeration, Optimization, Evolutionary Computation, Genetic Algorithm (GA), Particle Swarm Optimization (PSO), Simulated Annealing (SA)

\section{Model Validation and analysis of VCRS}

The optimization of a two-stage vapor compression refrigeration system uses the basis of design as per the published literature by Bakeem et al. [7]. Table 1 provides the condition for the basis of design.

Table 1: Condition for the base model

\begin{tabular}{lll}
\hline Process Parameter & Value & Unit \\
\hline Evaporator temperature & 0 & ${ }^{\circ} \mathrm{C}$ \\
Condenser temperature & 45 & ${ }^{\circ} \mathrm{C}$ \\
Outdoor temperature & 35 & ${ }^{\circ} \mathrm{C}$ \\
Indoor temperature & 25 & ${ }^{\circ} \mathrm{C}$ \\
Compressor efficiency & $91 \%$ & $\mathrm{Unitless}$ \\
Cooling load & 1 & $\mathrm{~kW}$ \\
Evaporator HFCs & 0.5 & $\mathrm{KWm}{ }^{-2} \mathrm{~K}^{-1}$ \\
Condenser HFCs & 0.5 & $\mathrm{KWm}^{-2} \mathrm{~K}^{-1}$ \\
Evaporator (Ammonia) & 1.1 & $\mathrm{KWm}^{-2} \mathrm{~K}^{-1}$ \\
Condenser (Ammonia) & 0.5 & $\mathrm{KWm}^{-2} \mathrm{~K}^{-1}$ \\
\hline
\end{tabular}

The optimization model also considers the desuperheating and subcooling effect by introducing a flash chamber in the intermediate pressure stage. As illustrated in Figure 1, refrigerant R134a leaves the evaporator as saturated vapor (point a) and then compressed to the intermediate pressure (point $b_{2}$ ) where it reaches the superheated vapor phase. Then the mixed desuperheated refrigerant went to the suction of the high stage compressor (point c) and compressed to high pressure (point $\mathrm{d}_{2}$ ), and the refrigerant goes to the superheated phase again. Here, mixed means the combined flow of the refrigerant from the outlet of the low stage compressor, and the refrigerant stream that is coming from the flash chamber.

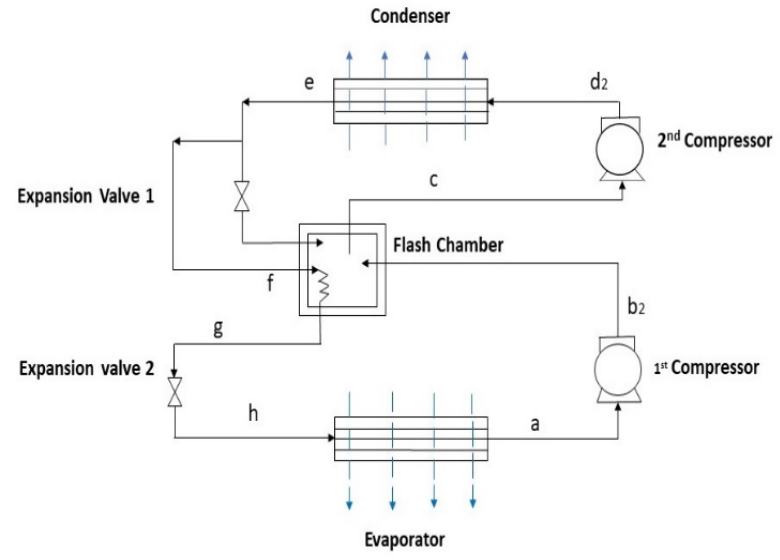

Figure 1. Basis of design for a two-stage vapor compression refrigeration system
The superheated refrigerant loses some enthalpies through the point to the condenser, and after entering to the heat exchanger of condenser, it cools down to saturated liquid (point e). At the exit point of the condenser (point e), the refrigerant splits into two streams. One stream enters an expansion valve (point $\mathrm{f}$ ) and then goes to the flash chamber. Another stream is subcooled first (point g) and then goes through an expansion valve. When the stream passes through the expansion valve, some part of the refrigerant will evaporate because of the throttling effect and vapor is produced (point h). The combined vapor and liquid streams of the refrigerant enter the evaporator where the refrigerant delivers the cooling, and the process continues its cycle. The basis of design is reproduced and validated by comparing the results from this work against the data from published literatures by Bakeem [7], Esfahani [8] and Gebreslassie [9]. In this work, the coefficient of performance "COP" and refrigerant mass flow ratio " $r$ " value for the basis of design is successfully validated with the results by Baakeem et al. [7] with the deviations in COP and refrigerant mass ratio at $3.28 \%$ and $0.89 \%$ respectively. The deviations are acceptably small and may be due to differences in refrigerant property tables used in this work.

\section{Formulation of optimization problem}

The modelling of a two-stage refrigeration system in this work follows the assumption that the

a) system is operating at steady state condition

b) kinetic and potential energy losses are negligible

c) pressure loss across the evaporator and condenser are negligible

d) refrigerant leaves the evaporator and condenser as saturated condition.

e) throttling process through expansion valve is isenthalpic

Mass balance:

$$
\sum m=0
$$

Energy balance:

$$
\sum Q+\sum W+\sum m h=0
$$

Heat transfer / cooling done by the evaporator:

$$
Q_{\text {evp }}=m_{\text {revp }} \times\left(h_{a}-h_{\mu p}\right)
$$

The refrigerant mass flow rate through the condenser is the summation of the mass flow rate through the evaporator and flash chamber. $m_{r, \text { cond }} ; m_{r, \text { evp }} ; m_{r, f c}$ Are the mass flow through the condenser, evaporator and flash chamber, respectively:

$$
m_{r, \text { cond }}=m_{r, \text { evp }}+m_{r, f c}
$$

Mass flow ratio is the ratio of refrigerant passing through the evaporator to the compressor, and it can be defined as: 


$$
r=\frac{m_{r, \text { evp }}}{m_{r, \text { cond }}}=\frac{\left(h_{b 2}-h_{e}\right)+a \times\left(h_{e}-h_{l}\right)}{\left(h_{b 2}-h_{e}\right)-b \times\left(h_{b 2}-h_{e}\right)}
$$

Subcooling parameter and desuperheating parameters can be defined from the general model developed by Torella et al. [6], where the subcooling parameter depends on the condenser outlet temperature, evaporator inlet temperature and the temperature of the saturated liquid on the saturation curve at the intermediate pressure. On the other hand, the desuperheating parameter depends on the exit temperature of the low stage compressor, the suction temperature of the second stage compressor and the temperature of the saturated vapor on the saturation curve.

$$
a=\frac{\left(h_{e}-h_{h}\right)}{\left(h_{e}-h_{l}\right)} ; b=\frac{\left(h_{b 2}-h_{c}\right)}{\left(h_{b 2}-h_{v}\right)}
$$

Work done by the low-pressure stage compressor

$$
W_{1}=m_{r, \text { evp }} \times\left(h_{b 2}-h_{a}\right)
$$

Work done by the high-pressure stage compressor

$$
W_{2}=m_{r, c o n} \times\left(h_{d 2}-h_{c}\right)
$$

The isentropic efficiency of the compressor, one and two, can be defined as:

$$
\begin{gathered}
\eta_{\text {is } 1}=\eta_{i s 2}=\frac{h_{b}-h_{a}}{h_{b 2}-h_{a}}=\frac{h_{d}-h_{c}}{h_{d 2}-h_{c}}=\eta_{\text {is }} \\
\eta_{\text {is }}=(0.85-0.04667) \times{ }^{p_{\text {out }}} / p_{\text {in }}
\end{gathered}
$$

Actual work done by the compressors are corrected against the total efficiency of the compressors, which is a product of electrical and mechanical efficiency. The amount of actual work done by the second compressor can be calculated in similar steps to equation (10), and the total amount of work by the two compressors is a combination of the actual work done by the individual compressor. Heat transfer through the condenser can be defined as

$$
Q_{\text {cond }}=m_{r, \text { cond }} \times\left(h_{d 2}-h_{e}\right)
$$

where $h_{d 2}$ and $h_{e}$ refer to the inlet and outlet enthalpies of the condenser. Coefficient of performance (COP) is the ratio of cooling duty by the evaporator to the actual work done by the two compressors, and it can be defined as:

$$
C O P=\frac{Q_{\text {evp }}}{W_{a c}}
$$

The optimization problem in this work first considers the minimization of energy consumption by the compressors according to the process equations outlined above and the range of operation, as shown in Table 2. The procedure is then repeated for maximization of COP.
During the simulation procedure, both evolutionary computations are repeated for three times each using different initial values for the populations, iteration and other specific properties.

\section{Solution procedures}

Evolutionary computation techniques are stochastic, population-based global search, optimization algorithms which works based on the inspiration of biological evolution. GA, PSO and SA are the three most widely used evolutionary computation technique. Figure 2 outlines the comparative algorithms for the evolutionary computational techniques among Genetic Algorithm (GA), Particle Swarm Optimization (PSO) and Simulated Annealing (SA).

\subsection{Particle Swarm Optimization (PSO)}

Particle swarm optimization is a stochastic, populationbased evolutionary technique. This technique has been successfully used to optimize various types of constrained and unconstrained, non-linear, multiobjective and non-differentiable functions [10]. It is a simulation algorithm based on the animal's social behavior, mainly birds flocking and fish schooling. In PSO a set of initial particle called swarm is generated randomly, each particle has it's own position and velocity, as the iteration starts the particles travel through the search space and look for the optimum solution, each particle of the swarm communicates with each other and update their position and velocity according to its own best (local best) and swarm's best (global best) position [17]. Here, each particle symbolizes a possible solution and a set of particles make up a swarm. When any of the swarm members find the optimum solution, others will follow it and converge to the optimum solution [18]. The search process will continue until a termination criterion has been met. PSO has a superior property that it can be directly used in continuous real number space and does not need the gradient value of the objective functions [11]. PSO needs less adjustment of parameters and can be easily implemented in various types of problems, it also has distinctive features of memory, needs low memory space and lesser rate of $\mathrm{CPU}$ which indicates better computational efficiency [15].

\subsection{Genetic Algorithm (GA)}

Genetic algorithm is a population-based evolutionary algorithm technique which has been generally used for various optimization and search problems [12]. GA uses a guided random search in a binary search space that generates many solutions to a problem, and the solutions are simultaneously refined to produce a set of solutions that are near optimum in a reasonable time [13]. This procedure works based on the natural processes of reproduction and selection in the population, in which each individual represents a possible solution [14]. 


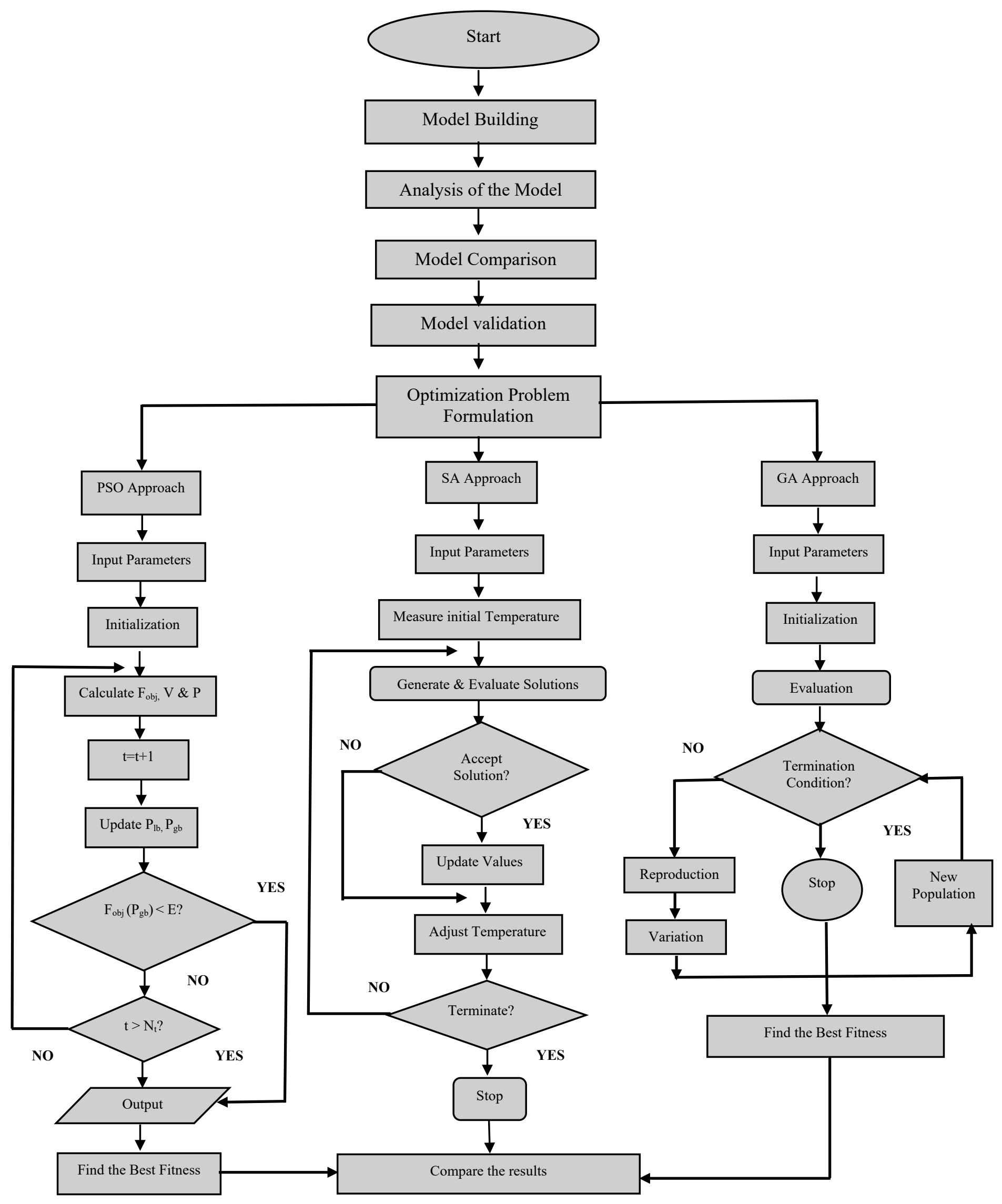

Figure 2: Flow chart of the working procedures 
Two basic reproduction and variation operation (crossover, mutation) has carried out continuously to appraise the populations until a termination criterion has been met. One iteration of operators is called a generation. As the solution representation is parallel to chromosomes and operators are alike to genetic operators, this process is named as genetic algorithm [16]. In this study, each individual represents the variable to be optimized, such as the refrigerant mass flow ratio, evaporator and condenser temperature, parameters ' $a$ ' and ' $b$ ' for sub-cooling and desuperheating.

Table 2: Comparison of the findings for optimal conditions of the base model:

\begin{tabular}{llll}
\hline $\begin{array}{l}\text { Parameter } \\
\text { name }\end{array}$ & $\begin{array}{l}\text { Findings by } \\
\text { Bakeem }\end{array}$ & $\begin{array}{l}\text { This } \\
\text { study }\end{array}$ & Difference \\
\hline COP & 3.61 & 3.72 & $3.28 \%$ \\
$\mathrm{r}$ & 1.232 & 1.243 & $0.89 \%$ \\
$\mathrm{a}$ & 1 & 1 & 0 \\
$\mathrm{~b}$ & 0 & 0 & 0 \\
\hline
\end{tabular}

\subsection{Simulated Annealing (SA)}

$\mathrm{SA}$ is a stochastic local search metaheuristic algorithm used to solve combinatorial optimization problems that allow continuous convergence to optimal solution [19, 20]. It consists of several uphill and downhill movement, which helps to move from an existing solution to a superior solution and increase exploration ability [20]. In addition to this, it also decreases the probability of getting stuck in a local solution by increasing variety in the domain [20]. SA tries to achieve the minimum energy status of any materials during simulation. It required to heat the process at an initial high temperature and decrease it quasistatically until any changes happened [21]. In case of stochastic combinatorial optimization problem, SA needs careful adjustment of the annealing schedule to get optimum performance of the algorithm, and the cooling schedule maintains the amount of iteration required to perform the simulation [22].

\section{Result and Discussion}

For the evolutionary computation of GA, PSO and SA, each computation technique is run for three times. Stable convergence is achieved for each run. Repeatability of the results ensures the robustness of the algorithms. Table 3 shows that the best fitness value for minimum energy consumption is $0.185 \mathrm{~kW}$, where the energy required for the base case is $0.268 \mathrm{~kW}$, resulting in almost $31 \%$ energy savings against the base case. Figures $\mathbf{3}$ and $\mathbf{4}$ further show that the fitness values are consistent for almost 70 iterations.
Table 3: Improvement of the base case by using GA \& PSO

\begin{tabular}{llllll}
\hline Name & $\begin{array}{l}\text { Base } \\
\text { case }\end{array}$ & \multicolumn{3}{c}{ Optimized Values } & Improvement \\
\hline & & GA & PSO & SA & \\
COP & 3.72 & 6.58 & 6.58 & 6.58 & $76.95 \%$ \\
Energy & 0.268 & 0.185 & 0.185 & 0.185 & $30.79 \%$ \\
r & 1.243 & 1.23 & 1.23 & 1.23 & \\
$\mathrm{a}$ & 1 & 1 & 1 & 1 & \\
$\mathrm{~b}$ & 0 & 0 & 0 & 0 & \\
$\mathrm{Te}$ & 0 & 10 & 10 & 10 & \\
$\mathrm{Tc}$ & 45 & 40 & 40 & 40 & \\
\hline
\end{tabular}

The optimum COP of 6.58 , compared to the base case COP of 3.72 , gives $77 \%$ better performance of the refrigeration system compared to the base case. The refrigerant mass flow ratio, $r$, sub-cooling parameter, a, and desuperheating parameter, $b$, remain unchanged because, during the basis of design, the flowsheet has been developed to achieve the best COP value at the initial level. This work also achieved a 3.28\% higher COP performance in comparison to the optimum results by Baakeem et al. [7] using the Conjugate Direction Method. An explicit comparison between the previous study done by Bakeem and the recent study has shown in table $\mathbf{2}$ and $\mathbf{5}$.

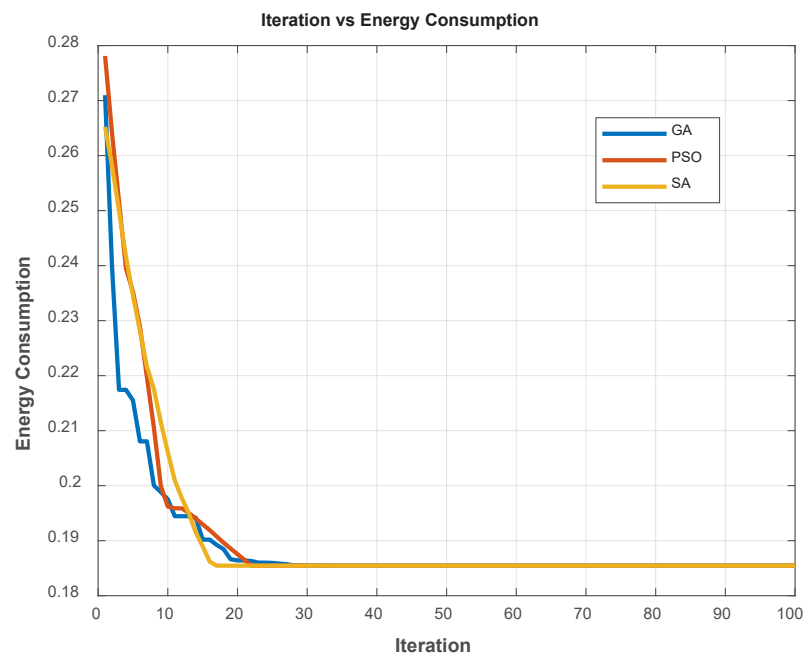

Figure 3: Convergence curve for minimization of the required Energy by GA and PSO algorithm.

Table 4 compares the performance of the three evolutionary computation techniques. The GA algorithm in this work uses random initialization within the lower and upper bound of the variables, while the reproduction probability is set at $80 \%$ by crossover and $30 \%$ by mutation. Reproduction increases the number of populations, which is then ranked for the selection according to the survival of the fittest principle and for the elimination of the extra populations produced from the reproduction of the populations. 


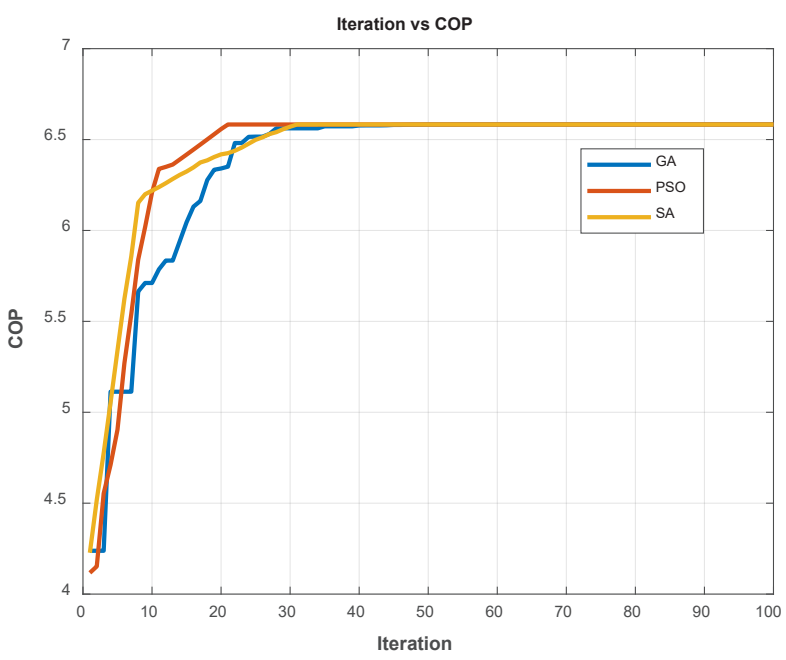

Figure 4: Convergence curve for the maximization of COP by GA and PSO algorithm.

Consequently, only the best/ elite populations at each iteration is stored for further generations. The reproduction strategy, such as crossover and mutation, maintains diversity and helps to ensure that the optimum results are obtained within a very short period of time. Furthermore, an extra range factor for the crossover (Gamma) was employed, and another mutation parameter (Sigma) was used to increase the exploitation range and exploration ability of the GA. During the optimization procedure, it was found that GA needs more population (50) to explore the search space for optimum values, and it converged comparatively later than PSO and SA (after 30 and 35 generation). However, for three consecutive runs, GA shows consistent optimization results, which indicates the robustness and proper convergence of the algorithm. On the other hand, the most significant property of PSO is the simplicity and easy programmability, requiring a smaller number of equations and input values as compared to GA. In PSO each particle has its own velocity and direction and the particles of the swarm communicate amongst themselves and update their position and velocity according to the neighbour/swarm's position and velocity. Each particle of the swarm has the general cognitive and social coefficient values, which helps to converge the whole swarm together to the optimum values. These properties make the algorithm simpler and easily usable. In addition to this the SA algorithm shows early convergence compared to GA and it requires almost same amount of simulation time and initial population as PSO. But, it needs more parameters to adjust to get the optimum results and proper convergence, which means it takes more computation effort to do the fine tuning of this algorithm. In case of convergence, we found that PSO, SA converged faster than GA and PSO required around 30\% less computational time than GA and SA. Here faster converge is a PSO's notable feature, and robustness is a noble features of GA. Overall, all of these 3 algorithms are designed properly to produce a good result along with perfect optimum convergence.

Table 4: Comparison of the performance of the Evolutionary Computation Techniques

\begin{tabular}{|c|c|c|c|c|c|c|}
\hline \multirow[t]{2}{*}{ Parameter of the Algorithm } & \multicolumn{2}{|c|}{ PSO } & \multicolumn{2}{|c|}{ GA } & \multicolumn{2}{|c|}{ SA } \\
\hline & Energy & $\mathrm{COP}$ & Energy & $\mathrm{COP}$ & Energy & $\mathrm{COP}$ \\
\hline Number of populations & 20 & 25 & 50 & 50 & 20 & 20 \\
\hline Iteration Number & 100 & 100 & 100 & 100 & 100 & 100 \\
\hline Sub-iteration number & -- & -- & -- & -- & 10 & 10 \\
\hline Total running time (Second) & 2.5 & 4 & 4 & 5 & 4 & 4.5 \\
\hline $\begin{array}{l}\text { Convergence starting point after (nth) } \\
\text { generation }\end{array}$ & 20 & 23 & 30 & 35 & 17 & 31 \\
\hline Cognitive constant $\left(\mathrm{C}_{1}\right)$ & 1.9 & 2 & -- & -- & -- & -- \\
\hline Social constant $\left(\mathrm{C}_{2}\right)$ & 1.8 & 1.9 & -- & -- & -- & -- \\
\hline Inertia Weight (w) & 1 & 1 & -- & -- & -- & -- \\
\hline Damping Ratio ( $\left.\mathrm{W}_{\text {damp }}\right)$ & 0.95 & 0.98 & -- & -- & -- & -- \\
\hline Crossover percentage & -- & -- & 0.8 & 0.8 & & \\
\hline Mutation percentage & -- & -- & 0.3 & 0.3 & 0.1 & 0.1 \\
\hline $\begin{array}{l}\text { Extra Range Factor for Crossover } \\
\text { (Gamma) }\end{array}$ & -- & -- & 0.4 & 0.4 & -- & -- \\
\hline Sigma & -- & -- & 0.1 & 0.1 & 0.1 & 0.1 \\
\hline alpha & -- & -- & -- & -- & 0.9 & 0.9 \\
\hline number of move & -- & -- & -- & -- & 4 & 4 \\
\hline initial temperature & -- & -- & -- & -- & 0.1 & 0.1 \\
\hline
\end{tabular}


Table 5: Comparison of optimization result

\begin{tabular}{lllclll}
\hline Optimized variables & Bakeem et al. [7] & & This Work & & Difference & Unit \\
\hline Optimization method & $\begin{array}{l}\text { Conjugate Direction } \\
\text { Method }\end{array}$ & GA & PSO & SA & & \\
COP & 6.01 & 6.5827 & 6.5828 & 6.5828 & $8.90 \%$ & --- \\
W & 0.17 & 0.1859 & 0.1854 & 0.1854 & $5.88 \%$ & KW \\
a & 1 & 1 & 1 & 1 & $0 \%$ & \\
b & 0 & 0 & 0 & 0 & $0 \%$ & \\
\hline
\end{tabular}

\section{Conclusion}

A two-stage VCRS has been analyzed and optimized using three evolutionary computation techniques. The results from this work show that form the basis of design, a two-stage VCRS can save 30.8\% energy, and the system performance may also be improved by $77 \%$. The three optimization algorithms, PSO, GA and SA performed better than the solution previously published in the literature using Conjugate direction Method, which is a direct search technique. During the simulation procedures, optimum results has been reached within 3-5 seconds of computation time. From the overall performance of the algorithm, it can be summarized that PSO is more straightforward, performs comparatively better than GA and $\mathrm{SA}$, it also requires less computational effort to do the fine tuning compared to the others.

\section{References}

1. BP Energy Outlook,

https://www.bp.com/content/dam/bp/businesssites/en/glo bal/corporate/pdfs/energy-economics/energy-outlook/bpenergyoutlook-2019.pdf [accessed 16th August 2019].

2. Gurubalana A, Maiyaa M P, Geoghegan P J, A comprehensive review of liquid desiccant air

conditioning system, Applied Energy 254 (2019) 113673.

3. Sun Y, Huang G, Li Z, Wang S, Multiplexed optimization for complex air conditioning systems, Building and Environment 65 (2013) 99-108.

4. Arshad M U, Ghani M U, Ullaha A, Gungor A, Zaman M, Thermodynamic analysis and optimization of double effect absorption refrigeration system using genetic algorithm, Energy Conversion and Management 192 (2019) 292-307.

5. Zhao L, Cai W, Ding X, Chang W, Model-based optimization for vapor compression refrigeration cycle, Energy 55 (2013) 392-402.

6. Torrella E, Larumbe J A,Cabello R, Llopis R, Sanchez D, Energy comparison of intermediate configurations in two-stage vapor compression refrigeration systems, Energy 36 (2011) 4119-4124.

7. Baakeem S S, Orfi J, Alabdulkarem A, Optimization of a multistage vapor-compression refrigeration system for various refrigerants, Applied Thermal Engineering 136 (2018) 84-96.
8. Esfahani I.J., Kang Y.T., Yoo C., A high efficient combined multi-effect evaporation absorption heat pump and vapor-compression refrigeration part 1, Energy $\mathbf{7 5}$ (2014) 312-326.

9. Gebreslassie B.H., Guillen-Gosalbez G., Jimenez L., Boer D, Design of environmentally conscious absorption cooling systems via multi-objective optimization and life cycle assessment, Appl. Energy 86 (2009) 1712-1722.

10. J. Kennedy, R. Eberhart, Particle swarm optimization, in: Proceedings of the 1995 IEEE International Conference on Neural Networks, vol. 4, 1995, pp. 1942-1948.

11. Khare A, Rangnekar S, A review of particle swarm optimization and its applications in Solar Photovoltaic system, Applied Soft Computing 13 (2013) 2997-3006.

12. Mitchell, Melanie (1996). An Introduction to Genetic Algorithms. Cambridge, MA: MIT Press. ISBN 9780585030944.

13. Deb K, Practical Optimization Using Evolutionary Methods, KanGAL Report Number 2005008, http://www.iitk.ac.in/kangal

14. Michalewicz Z., Genetic Algorithms, Springer Verlag, 1995.

15. She X, Cong L, Nie B, Leng G, Peng H, Chen Y, Zhang X, Wen T, Yang H, Luo, Y, Energy-efficient and economic technologies for air conditioning with vapor compression refrigeration: A comprehensive review, Applied Energy 232 (2018) 157-186

16. Deb K, Practical Optimization Using Evolutionary Methods, KanGAL Report Number 2005008, http://www.iitk.ac.in/kangal

17. J. Kennedy, R. Eberhart, Particle swarm optimization, in: Proceedings of the 1995 IEEE International Conference on Neural Networks, vol. 4, 1995, pp. 1942-1948.

18. Marini F, Walczak B, Particle swarm optimization (PSO). A tutorial, Chemometrics and Intelligent Laboratory Systems 149 (2015) 153-165.

19. S. Kirkpatrick, C.D. Gelatt, M.P. Vecchi, Science 220 (4598) (1983) 671.

20. Amine K, Multiobjective Simulated Annealing:

Principles and Algorithm Variants, Advances in

Operations Research,

https://doi.org/10.1155/2019/8134674

21. Vicente J D, Lanchares J, Hermida R, Placement by thermodynamic simulated annealing, Physics Letters A 317 (2003) 415-423, doi:

10.1016/j.physleta.2003.08.070

22. K.H. Hoffmann, A. Franz, P. Salamon, Phys. Rev. E 66 (2002) 046706. 